Clinical consequences of relative biological effectiveness variations in proton radiotherapy of the prostate, brain and liver

This article has been downloaded from IOPscience. Please scroll down to see the full text article.

2013 Phys. Med. Biol. 582103

(http://iopscience.iop.org/0031-9155/58/7/2103)

View the table of contents for this issue, or go to the journal homepage for more

Download details:

IP Address: 157.193.1.253

The article was downloaded on 24/06/2013 at 22:46

Please note that terms and conditions apply. 


\title{
Clinical consequences of relative biological effectiveness variations in proton radiotherapy of the prostate, brain and liver
}

\author{
Alejandro Carabe $^{1}$, Samuel España ${ }^{2}$, Clemens Grassberger ${ }^{3}$ \\ and Harald Paganetti ${ }^{3}$ \\ ${ }^{1}$ Department of Radiation Oncology, University of Pennsylvania, Philadelphia, PA19104, USA \\ 2 Department of Medical Imaging and Signal Processing (MEDISIP), University of Ghent, \\ 9000 Ghent, Belgium \\ ${ }^{3}$ Department of Radiation Oncology, Massachusetts General Hospital and Harvard Medical \\ School, Boston, MA02114, USA \\ E-mail: a.carabe@uphs.upenn.edu
}

Received 6 June 2012, in final form 13 January 2013

Published 8 March 2013

Online at stacks.iop.org/PMB/58/2103

\begin{abstract}
Proton relative biological effectiveness (RBE) is known to depend on the $(\alpha / \beta)_{x}$ of irradiated tissues, with evidence of $\sim 60 \%$ variation over $(\alpha / \beta)_{x}$ values from $1-10 \mathrm{~Gy}$. The range of $(\alpha / \beta)_{x}$ values reported for prostate tumors $(1.2-$ $5.0 \mathrm{~Gy})$, brain tumors (10-15 Gy) and liver tumors (13-17 Gy) imply that the proton $\mathrm{RBE}$ for these tissues could vary significantly compared to the commonly used generic value of 1.1 . Our aim is to evaluate the impact of this uncertainty on the proton dose in Gy(RBE) absorbed in normal and tumor tissues. This evaluation was performed for standard and hypofractionated regimens. RBE-weighted total dose (RWTD) distributions for 15 patients (five prostate tumors, five brain tumors and five liver tumors) were calculated using an in-house developed RBE model as a function of dose, dose-averaged linear energy transfer $\left(\mathrm{LET}_{d}\right)$ and $(\alpha / \beta)_{x}$. Variations of the dose-volume histograms (DVHs) for the gross tumor volume (GTV) and the organs at risk due to changes of $(\alpha / \beta)_{x}$ and fractionation regimen were calculated and the RWTD received by $10 \%$ and $90 \%$ of the organ volume reported. The goodness of the plan, bearing the uncertainties, was then evaluated compared to the delivered plan, which considers a constant RBE of 1.1. For standard fractionated regimens, the prostate tumors, liver tumors and all critical structures in the brain showed typically larger RBE values than 1.1. However, in hypofractionated regimens lower values of $\mathrm{RBE}$ than 1.1 were observed in most cases. Based on DVH analysis we found that the RBE variations were clinically significant in particular for the prostate GTV and the critical structures in the brain. Despite the uncertainties in the biological input parameters when estimating RBE values, the results show that the use of a variable RBE with dose, $\mathrm{LET}_{d}$ and $(\alpha / \beta)_{x}$ could help to further optimize the target dose in proton treatment
\end{abstract}


planning. Most importantly, this study shows that the consideration of RBE variations could influence the comparison of proton and photon treatments in clinical trials, in particular in the case of the prostate.

(Some figures may appear in colour only in the online journal)

\section{Introduction}

The relative biological effectiveness (RBE) of protons in radiotherapy is known to depend (among other factors) on the dose per fraction, the dose-averaged linear energy transfer $\left(\mathrm{LET}_{d}\right)$ distribution and the tissue $\alpha / \beta$ ratio obtained from cell survival curves after exposure to X-rays $\left((\alpha / \beta)_{x}\right)$. For proton dose distributions in a patient, LET $_{d}$ values can be up to six times larger than in a therapeutic X-ray beam $\left(110 \mathrm{MeV}\right.$ protons with $\mathrm{LET}_{d} \leqslant 12 \mathrm{keV} \mu \mathrm{m}^{-1}$ (Grassberger et al 2011) compared to electrons with $\mathrm{LET}_{d} \leqslant 2 \mathrm{keV} \mu \mathrm{m}^{-1}$ produced from $10 \mathrm{MV}$ photons (Amols and Kliauga 1985)). Recent results show considerable variations of $\mathrm{LET}_{d}$ values within the irradiated volume in proton therapy (Grassberger et al 2011), which in combination with findings on a dependence of RBE with tissue type (Gerweck and Kozin 1999) could imply a departure of RBE from the average value of 1.1 for certain tissues or treatment scenarios. Also, with the current tendency toward hypofractionated regimens in particle radiotherapy the application of a generic RBE for all treatments might have to be revisited, especially since there is very limited data from hypofractionated proton treatments.

This work aims at quantifying the theoretical departure of RBE from the accepted value of 1.1 (Paganetti et al 2002) based on a previously published RBE model (Carabe-Fernandez et al 2007) when the variability of the tissue $(\alpha / \beta)_{x}$ is considered and the dose per fraction is increased. Previous published work has been focused mainly on variations of RBE due to changes of $\mathrm{LET}_{d}$ (Grassberger et al 2011, Wilkens and Oelfke 2004, Tilly et al 2005, Frese et al 2011), whereas this work analyzes in addition variations due to changes in $(\alpha / \beta)_{x}$ and dose per fraction. This analysis will be presented for the treatment of prostate, brain and liver with proton therapy and compared with the dose-volume histograms (DVH) obtained from biological dose distributions assuming a constant RBE.

\section{Methods}

We apply a model (Carabe-Fernandez et al 2007, 2010) that establishes the RBE dependence with proton dose $\left(d_{p}\right), \operatorname{LET}_{d}$ and $(\alpha / \beta)_{x}$ (equation (1)):

$\mathrm{RBE}\left[\mathrm{LET}_{d}, d_{p},(\alpha / \beta)_{x}\right]=\frac{\sqrt{(\alpha / \beta)_{x}^{2}+4(\alpha / \beta)_{x} \mathrm{RBE}_{\max }\left[\mathrm{LET}_{d},(\alpha / \beta)_{x}\right] d_{p}+4\left(\mathrm{RBE}_{\min }\left[\mathrm{LET}_{d},(\alpha / \beta)_{x}\right]\right)^{2} d_{p}^{2}-(\alpha / \beta)_{x}}}{2 d_{p}}$,

where $\mathrm{RBE}_{\max }=\alpha_{p} / \alpha_{x}$ and $\mathrm{RBE}_{\min }=\sqrt{\beta_{p} / \beta_{x}}$ with $\left(\alpha_{p}, \beta_{p}\right)$ representing the linear-quadratic (LQ) parameters of cell survival curves for protons and $\left(\alpha_{x}, \beta_{x}\right)$ are the corresponding values for $\mathrm{x}$-rays. $\mathrm{RBE}_{\max }$ and $\mathrm{RBE}_{\min }$ are the asymptotic values of $\mathrm{RBE}$ at proton doses of 0 and $\infty \mathrm{Gy}$, respectively. In this work, the base values of $\mathrm{RBE}_{\max }$ and $\mathrm{RBE}_{\min }$ are derived from values of $\alpha$ and $\beta$ obtained from single dose survival curves of V79 cell cultures exposed to $\mathrm{x}$-rays and protons. However, the values of $\alpha$ and $\beta$ for the cell lines of the tissues involved in the treatment of the prostate, brain or liver, exposed to protons or x-rays are different. The impact of those differences on the model predictions for $\mathrm{RBE}_{\max }$ and $\mathrm{RBE}_{\min }$ have been incorporated using assumptions on how $\mathrm{RBE}_{\max }$ and $\mathrm{RBE}_{\min }$ change with tissue $(\alpha / \beta)_{x}$. There 
are considerable uncertainties when analyzing $\mathrm{RBE}_{\max }$ and $\mathrm{RBE}_{\min }$ as a function of $\alpha / \beta$. Based on the limited data available, this uncertainty seems to be larger for $\mathrm{RBE}_{\min }$. It has been suggested that $\mathrm{RBE}_{\min }$ could take values larger as well as smaller than unity (Daşu 2007, Carabe-Fernandez et al 2007).

The details explaining how RBE changes with $\mathrm{LET}_{d}$ and $(\alpha / \beta)_{x}$ are given in a previous publication (Carabe et al 2012). In general, experimental data on proton RBE versus $\mathrm{LET}_{d}$ (Folkard et al 1996, Belli et al 1989, 1993, Perris et al 1986, Coutrakon et al 1997, Wouters et al 1996, Schettino et al 2001) and proton RBE versus $(\alpha / \beta)_{x}$ (Gerweck and Kozin 1999, Paganetti et al 2000) indicate that RBE increases with increasing $\mathrm{LET}_{d}$ (note that for protons the eventual decrease with $\mathrm{LET}_{d}$ happens at $\mathrm{LET}_{d}$ values irrelevant for clinical considerations) but decreases with increasing $(\alpha / \beta)_{x}$. The current data sets used to relate RBE with $\mathrm{LET}_{d}$ and $(\alpha / \beta)_{x}$ are subject to uncertainties (Carabe et al 2012, Carabe 2013).

(i) $\mathrm{The}_{\mathrm{LET}}$ distribution within a proton beam depends on the range (i.e. nominal energy of the beam) and width of the spread out Bragg peak (SOBP). In particular, the larger the range the lower the $\mathrm{LET}_{d}$ values observed at any depth within the beam, and the larger the width of the SOBP, the lower the $\mathrm{LET}_{d}$ at the center of the SOBP. This complicates the interpretation of the data used to establish the relationship between proton RBE and $(\alpha / \beta)_{x}$ (Gerweck and Kozin 1999) as it was measured at the center of the SOBP using beams of different energies and with different SOBP widths.

(ii) If RBE is assumed to change with $(\alpha / \beta)_{x}$, then a new relationship between RBE and $\mathrm{LET}_{d}$ is required for every cell line or tissue type. The lack of data on RBE versus $\mathrm{LET}_{d}$ for any other cell line implies that we can only estimate how RBE relates to $\mathrm{LET}_{d}$ for cell lines with different $(\alpha / \beta)_{x}$. Equation (1) changes with $\mathrm{LET}_{d}$ through $\mathrm{RBE}_{\max }$ and $\mathrm{RBE}_{\min }$ and equations (2) and (3) show how the slope of the linear relationship between $\mathrm{RBE}_{\max }$ and $\mathrm{RBE}_{\text {min }}$ with $\mathrm{LET}_{d}$ changes with $(\alpha / \beta)_{x}$. Equations (2) and (3) assume that $\mathrm{RBE}_{\max }$ and $\mathrm{RBE}_{\text {min }}$ change with $(\alpha / \beta)_{x}$ and LET obeying a similar relationship, i.e. reciprocal to $(\alpha / \beta)_{x}$ and directly proportional to LET. However there is not sufficient data to confirm the validity of such an assumption. An analysis of neutron data shows a possible different relationship of $\mathrm{RBE}_{\min }$ and $(\alpha / \beta)_{x}$ (Jones et al 2011). According to a previous study the uncertainty in $(\alpha / \beta)_{x}$ translates into the uncertainty of $\mathrm{RBE}_{\max }$ and $\mathrm{RBE}_{\min }$ (Carabe et al 2012):

$$
\begin{aligned}
& \operatorname{RBE}_{\text {max }}\left[\operatorname{LET}_{d},(\alpha / \beta)_{x}\right]=0.843+0.154 \frac{2.686}{(\alpha / \beta)_{x}} \mathrm{LET}_{d}, \\
& \mathrm{RBE}_{\text {min }}\left[\mathrm{LET}_{d},(\alpha / \beta)_{x}\right]=1.09+0.006 \frac{2.686}{(\alpha / \beta)_{x}} \mathrm{LET}_{d} .
\end{aligned}
$$

The RBE-weighted DVHs presented in the results are solely originated by the variation of the slope of equations (2) and (3) due to the reported uncertainty range of the $(\alpha / \beta)_{x}$ values of the prostate, brain and liver.

\section{The dependence of biological dose distributions on dose per fraction}

In order to calculate the total doses relevant to the hypofractionated regimes, we have followed the biological effective dose (BED) calculation guidelines recommended by The Royal College of Radiologists (2008) and those recommendations by Dale et al (2002). In these guidelines the strategy suggested for the BED calculation is to calculate the total dose required to produce the same effect in the late-normal tissue and then to subsequently assess the likely effect of the changed schedule on the tumor, rather than the other way around. This is specifically recommended for the case of moving to a schedule with a smaller number of 
fractions, as in this case there is a real danger that the tolerance dose to the late-normal tissue might be exceeded. Therefore, the resultant total hypofractionated doses were: $\mathrm{TD}_{\mathrm{Brain}}=47.6$ $\mathrm{Gy}, \mathrm{TD}_{\text {Liver }}=51.5 \mathrm{~Gy}$ and $\mathrm{TD}_{\text {Prostate }}=49.3 \mathrm{~Gy}$ for 10 fractions; and $\mathrm{TD}_{\text {Brain }}=31.4 \mathrm{~Gy}$, $\mathrm{TD}_{\text {Liver }}=34.4 \mathrm{~Gy}$ and $\mathrm{TD}_{\text {Prostate }}=30 \mathrm{~Gy}$ for 3 fractions. For instance, in the case of prostate ( + seminal vesicles) cases, the calculation of a hypofractionated treatment (3 or 10 fractions) that would induce the same level of toxicity as the standard fractionation scheme ( 39 fractions) would be performed as

$$
78 \text { (Gy) }\left(1+\frac{2(\mathrm{~Gy})}{3(\mathrm{~Gy})}\right)=10 \mathrm{~d}\left(1+\frac{\mathrm{d}}{3(\mathrm{~Gy})}\right) \Rightarrow \mathrm{d}=4.93(\mathrm{~Gy}),
$$

where 78 Gy corresponds to the prescribed treatment dose, 2 Gy is the dose per fraction and 3 Gy corresponds to the assumed $(\alpha / \beta)_{x}$ of the normal tissue involved in the treatment. The fact that we are performing this calculation using a 1 within the brackets (i.e. ... $(1+\ldots)$ ) implies that this calculation is performed to obtain isoeffectiveness when comparing two photon treatments using different number of fractions.

There were cases where the treatment target overlapped with a critical structure. In these cases, the $\alpha / \beta$ used for the RBE calculation in the overlapping volume was the one from the critical structure. It was assumed that these volumes would be constituted by both normal and tumor cells, so a BED calculation that provides the total dose to deliver in a hypofractionated treatment that produces the same effect in the late-normal tissue should preserve the effect on the cells within the overlapping volume.

\section{Calculation of the dose, $L E T_{d}$ and RBE-weighted dose distributions}

The proton dose and $\mathrm{LET}_{d}$ distributions within the patients were calculated using Monte Carlo simulations (Grassberger et al 2011, Paganetti et al 2008). The 'variable' RBE distributions were calculated through equation (1) using the proton dose (normalized to the relevant dose per fraction obtained from the BED calculation) and $\mathrm{LET}_{d}$ distributions as well as assigned values of $(\alpha / \beta)_{x}$ to the different regions of interest (ROIs) involved in each plan. Figure 1 shows the dose distribution for one of the patients in each treatment site to provide an idea of the location of the diseases being considered. The RBE-weighted dose per fraction was multiplied by the number of fractions in order to obtain the RBE-weighted total dose (RWTD) for the conventional and hypofractionated plans $(\mathrm{RBE} \times$ Proton dose per fraction $\times$ number of fractions). Table 1 summarizes the relevant radiobiological parameters as well as the prescribed total doses currently used in this study for the treatment of the prostate gland, craniopharyngioma and hepato-cellular carcinoma. These total doses are prescribed for the treatment for either photon or proton treatments, but when protons are used the delivered dose is divided by a factor of 1.1 to account for the clinical RBE.

\section{Calculation of RWTD ranges and their uncertainty due to patient variability}

No specific criterion was used to select the five patients in each site except for the similarity in shape and location of the tumors in order to reduce the difference on the DVHs among each patient group. The upper and lower limit of the RWTD ranges in table 2 are obtained as an average of the RWTD for each patient in each site when the RBE is calculated according to equation (1) using the respective lower and upper limit of $(\alpha / \beta)_{x}$ indicated on the top of the table for each tissue. Therefore, each of the numbers in table 2 is subject to a variance, which is reported in table 3 (only for the standard fractionation when $\mathrm{RBE} \neq 1$ ). For instance, the upper 

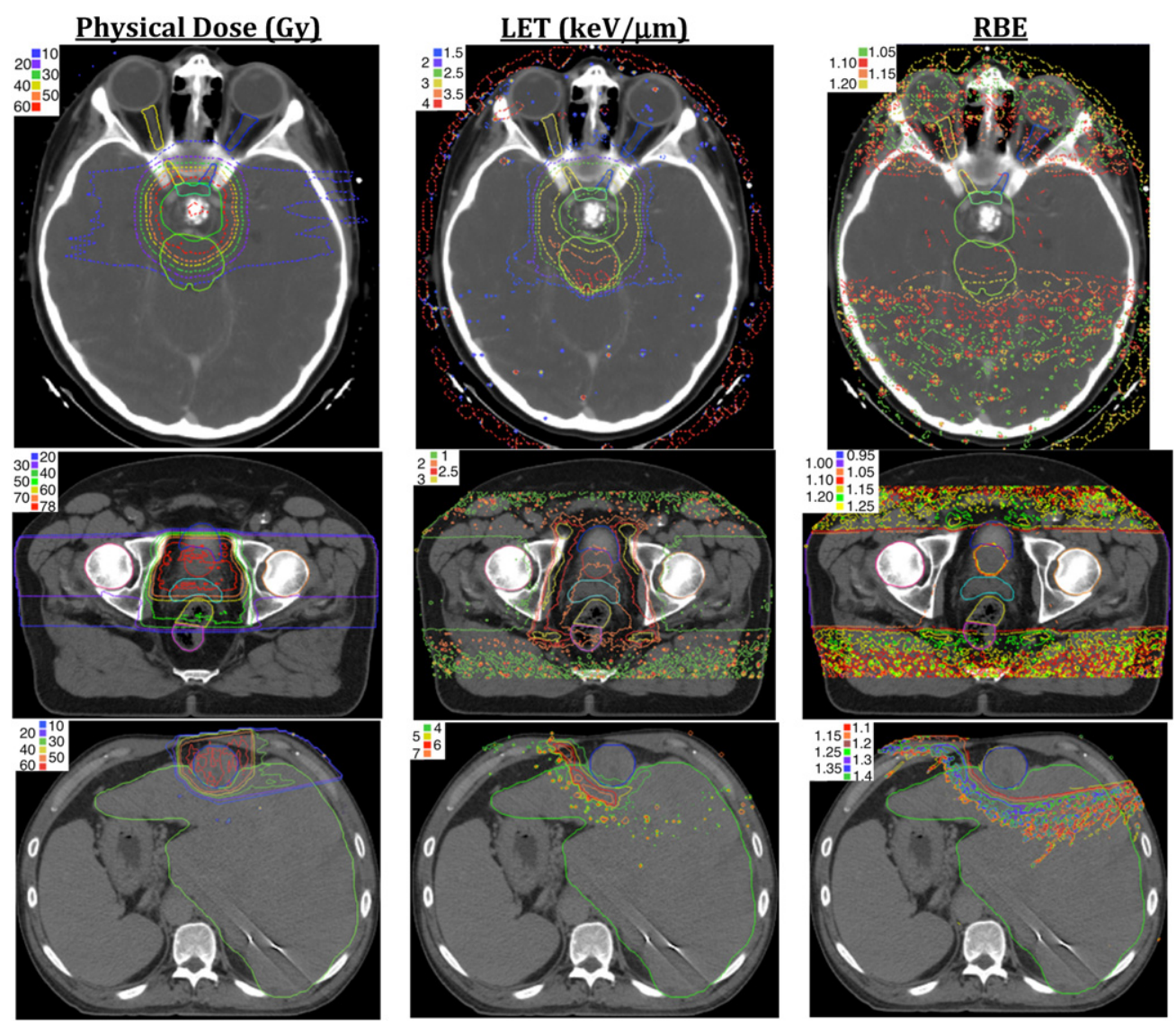

Figure 1. Representative distributions of physical dose, LET and RBE found in the brain, liver and prostate treatment plans. The dose cutoff used to calculate the dose average LET distributions was arbitrarily set at $0.1 \%$ of the target dose, which explains the noisiness of the distributions.

bound of the RWTD10\% (when RBE $\neq 1.1$ ) for the left optical nerve, i.e. 41 Gy(RWTD) ${ }^{4}$, is derived as the average of the RWTD10\% for each of the five brain patients $(50.256,30.678$, 25.667, 47.059, $51.989 \mathrm{~Gy}(\mathrm{RWTD})$ ). The resultant standard deviation (SD) in this case is 12.090 Gy(RWTD), as shown in table 3 .

\section{Results}

Table 2 shows the numerical results of this analysis. The number in brackets indicates the range of the RWTDs for each tissue at $10 \%$ and $90 \%$ of the organ volume. As expected these ranges are always larger for the standard fractionation schemes with lower doses per fractions.

As each value in table 2 represents an average of the RWTDs obtained for each patient of the five patients studied in each site, they have an uncertainty presented in table 3 in the form of 1 SD. Only the SDs for the RWTD calculated with RBE $\neq 1$ in standard fractionations have

4 The standard unit for the RBE-weighted dose is Gy(RBE). In this study, dose is weighted with either a constant $(\mathrm{RBE}=1.1)$ or a variable $(\mathrm{RBE} \neq 1.1) \mathrm{RBE}$, so in order to distinguish how the RBE-weighted doses reported in this study are obtained, we have used $\mathrm{Gy}(\mathrm{RBE})$ when $\mathrm{RBE}=1.1$ and $\mathrm{Gy}(\mathrm{RWTD})$ when $\mathrm{RBE}$ is considered to vary with dose, LET or $(\alpha / \beta)_{x}$. 
Table 1. Target prescribed doses and dose constrains to the normal tissues used in this study for prostate carcinomas, craniopharyngiomas and hepato-cellular carcinomas. The footnotes indicate the literature source of the $(\alpha / \beta)_{x}$.

\begin{tabular}{|c|c|c|}
\hline & $\begin{array}{l}\text { Prescribed dose and critical } \\
\text { structure dose constrains }\end{array}$ & $\alpha / \beta(95 \% \mathrm{CI})$ \\
\hline \multicolumn{3}{|c|}{ Prostate gland (standard number of fractions $=39$ ) } \\
\hline GTV + seminal vesicles & $78 \mathrm{~Gy}$ & $\begin{array}{l}\text { GTV: } 1.5^{\mathrm{a}}\left(1.2^{\mathrm{b}}, 5.6^{\mathrm{c}}\right) \\
\text { SEM V.: } 3.0(2.5,3.5)\end{array}$ \\
\hline Rectum & $\mathrm{V} 75<10 \%, \mathrm{~V} 70<70 \%$ & $4.0^{\mathrm{d}}(2.5,5.0)^{\mathrm{e}}$ \\
\hline Bladder & & $4.0^{\mathrm{d}}(3.0,7.0)^{\mathrm{e}}$ \\
\hline \multicolumn{3}{|c|}{ Craniopharyngioma (standard number of fractions $=29$ ) } \\
\hline GTV & 72 Gy (x-rays + proton boost) & $12^{\mathrm{f}}(10,15)^{\mathrm{f}}$ \\
\hline Brainstem & No hot spot & $2.1^{\mathrm{g}}(1.5,3.9)^{\mathrm{g}}$ \\
\hline Chiasm & No hot spot & $2.9^{\mathrm{i}}(1.5,3.9)^{\mathrm{g}}$ \\
\hline Optical nerve & No hot spot (56-60 Gy) & $1.6^{\mathrm{h}}(0.5,10.3)^{\mathrm{h}}$ \\
\hline Normal brain & & $2.9^{\mathrm{i}}(1.5,3.9)^{\mathrm{g}}$ \\
\hline \multicolumn{3}{|c|}{ Hepato-cellular carcinoma (standard number of fractions $=15$ ) } \\
\hline GTV & $60 \mathrm{~Gy}$ & $15.0^{j}(13.0,17.0)^{j}$ \\
\hline Lung & $\begin{array}{l}\text { EUD }<20 \mathrm{~Gy} \text {; or mean dose } \\
<24 \text { Gy (whichever lowest) } / 4 \mathrm{~Gy}\end{array}$ & $4.4^{\mathrm{k}}(1.5,6.9)^{\mathrm{k}}$ \\
\hline Healthy liver & No hot spot & $2.00^{\mathrm{j}}(1.5,3.00)$ \\
\hline
\end{tabular}

a Brenner and Hall (1999).

${ }^{\mathrm{b}}$ Ritter et al (2009).

c Daşu (2007).

${ }^{\mathrm{d}}$ Koukourakis et al (2007).

e Fowler (2005).

f Yuan et al (2008).

${ }^{g}$ Meeks et al (2000).

$\mathrm{h}$ Jiang et al (1994).

${ }^{\mathrm{i}}$ Lawrence et al (2010).

$\mathrm{j}$ Tai et al (2008).

${ }^{\mathrm{k}}$ Fowler (1989).

The CI of the $(\alpha / \beta)_{x}$ are also included.

been included in table 3. In this table the 'Upper' and 'Lower' labels refer to SD of the upper and lower values of the RWTD ranges presented in each cell of table 2 obtained for the lower and upper values, respectively, of $(\alpha / \beta)_{x}$ for each tissue. These SDs should be interpreted as the uncertainty of the upper, central and lower lines of the biological DVHs in figures 2 and 3 (left panels) purely related to patient variability and not due to $(\alpha / \beta)_{x}$ variability. According to this table, the SDs of the RWTDs in the target volumes are small (1-6 Gy(RWTD)) in the gross tumor volumes (GTVs) but larger SDs are observed in the large dose region (i.e. $10 \%$ volumes) in normal tissues, especially when the volume of the organ is small such as the chiasm and optical nerve. There is a larger SD for lower $(\alpha / \beta)_{x}$.

Figures 2 and 3 show, on the left, the resultant biological DVH (RWTD-VH) for the biological doses in the GTV and critical structures considering RBE $=1.1$ compared to that obtained when RBE is assumed dependent on dose, $\mathrm{LET}_{d}$ and $(\alpha / \beta)_{x}$. To limit the number of figures, only the RWTD-VHs for prostate and brain are shown as the effects found for the liver cases were smaller. The shaded areas correspond to the RWTD obtained from the $\mathrm{RBE}$ calculated from the top and bottom values of the $(\alpha / \beta)_{x}$ confidence interval $(\mathrm{CI})$, i.e. the shaded areas solely correspond to the uncertainties in $(\alpha / \beta)_{x}$. Because it is difficult to separate the influence from the $\mathrm{LET}_{d}$ and the $(\alpha / \beta)_{x}$ on the RBE, we have included the $\mathrm{LET}_{d^{-}}$and RBEvolume-histograms within each ROI for the nominal (central) values of $(\alpha / \beta)_{x}$ in figures 2 and 3. For example, the $\mathrm{LET}_{d}$-volume-histograms for the prostate GTV and the seminal vesicles 
Table 2. Average RWTDs received by $10 \%$ and $90 \%$ of the GTV and organ-at-risk volumes in prostate, brain and liver proton therapy (five patients each). The number in brackets in each cell represents the RWTD range when RBE $\neq 1.1$. ' $n$ ' represents the number of fractions. The units of the RWTD obtained from variable RBE (RBE $\neq 1.1)$ is Gy(RWTD), while hat obtained from a constant RBE $(\mathrm{RBE}=1.1)$ is measured in $\mathrm{Gy}(\mathrm{RBE})$.

\begin{tabular}{|c|c|c|c|c|c|c|c|c|c|c|c|c|c|}
\hline & & & 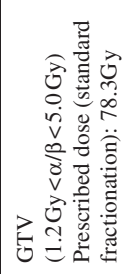 & 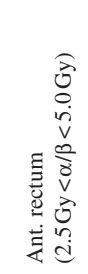 & 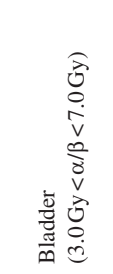 & 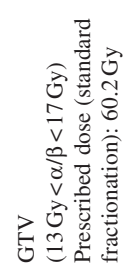 & 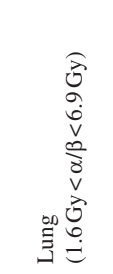 & 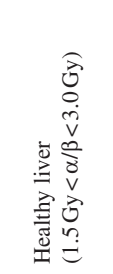 & 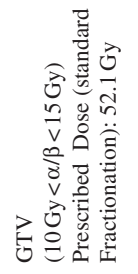 & 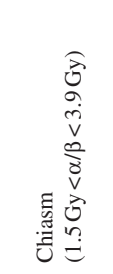 & 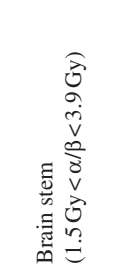 & 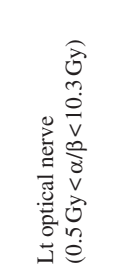 & 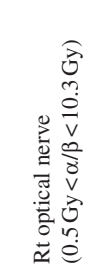 \\
\hline \multirow{2}{*}{ II } & RWTD10 & $\begin{array}{l}\mathrm{RBE} \neq 1.1 \\
\mathrm{RBE}=1.1\end{array}$ & $\begin{array}{c}36-49 \\
(13) \\
35.5\end{array}$ & $\begin{array}{l}31-32 \\
(1) \\
33.9\end{array}$ & $\begin{array}{c}29.6-29.8 \\
(0.2) \\
32.4\end{array}$ & $\begin{array}{c}30.9-31.3 \\
(0.4) \\
32.4\end{array}$ & $\begin{array}{c}14.6-14.8 \\
(0.2) \\
15.8\end{array}$ & $\begin{array}{c}11.6-11.8 \\
(0.2) \\
12.3\end{array}$ & $\begin{array}{c}31.7-32.6 \\
(0.9) \\
33.7\end{array}$ & $\begin{array}{c}30.7-33.5 \\
(2.8) \\
33.6\end{array}$ & $\begin{array}{c}29.8-32.8 \\
(3) \\
32.5\end{array}$ & $\begin{array}{c}23.4-26.7 \\
(3.3) \\
25.58\end{array}$ & $\begin{array}{c}22.0-25.6 \\
(3.6) \\
24.5\end{array}$ \\
\hline & RWTD90 & $\mathrm{RBE} \neq 1.1$ & $\begin{array}{c}35-47 \\
(12)\end{array}$ & $\begin{array}{l}8-9 \\
\text { (1) }\end{array}$ & 0.1 & $\begin{array}{c}29.2-29.6 \\
(0.4)\end{array}$ & 0.01 & 0 & $\begin{array}{c}29.9-32 \\
(2.1)\end{array}$ & $\begin{array}{c}29.8-32.6 \\
(2.8)\end{array}$ & $\begin{array}{c}0.02-0.03 \\
(0.01)\end{array}$ & $\begin{array}{c}0.08-0.13 \\
(0.05)\end{array}$ & $\begin{array}{c}0.08-013 \\
(0.05)\end{array}$ \\
\hline \multirow{2}{*}{$\stackrel{\circ}{=}$} & RWTD10 & $\begin{array}{l}\mathrm{RBE}=1.1 \\
\mathrm{RBE} \neq 1.1 \\
\mathrm{RBE}=1.1\end{array}$ & $\begin{array}{c}57-80 \\
(23) \\
55.5 \\
\end{array}$ & $\begin{array}{c}9.1 \\
49-50 \\
(1) \\
52.9 \\
\end{array}$ & $\begin{array}{c}0.12 \\
46-47 \\
(1) \\
50.5 \\
\end{array}$ & $\begin{array}{c}50.0 \\
48.49 .4 \\
(0.8) \\
50.5 \\
\end{array}$ & $\begin{array}{c}0.01 \\
23.1-23.5 \\
(0.4) \\
24.6 \\
\end{array}$ & $\begin{array}{c}0 \\
18.6-19.8 \\
(1.2) \\
19.2\end{array}$ & $\begin{array}{c}32.3 \\
52.3-58.3 \\
(6) \\
55.1\end{array}$ & $\begin{array}{c}32.6 \\
50.2-58.3 \\
(8.1) \\
54.8\end{array}$ & $\begin{array}{c}0.03 \\
48.9-57.4 \\
(8.5) \\
53.0\end{array}$ & $\begin{array}{c}0.08 \\
39-48 \\
(9.0) \\
41.8\end{array}$ & $\begin{array}{c}0.08 \\
36-46 \\
(10) \\
39.9\end{array}$ \\
\hline & RWTD90 & $\begin{array}{l}\mathrm{RBE} \neq 1.1 \\
\mathrm{RBE}=1.1\end{array}$ & $\begin{array}{c}56-77 \\
(21) \\
53.8\end{array}$ & $\begin{array}{c}13-14 \\
(1) \\
14.1\end{array}$ & $\begin{array}{l}0.20 \\
0.20\end{array}$ & $\begin{array}{c}45.8-46.7 \\
(0.9) \\
47.6\end{array}$ & $\begin{array}{l}0.01 \\
0.01\end{array}$ & $\begin{array}{l}0.01 \\
0.01\end{array}$ & $\begin{array}{c}49-53.2 \\
(4.2)\end{array}$ & $\begin{array}{c}48.8-56.6 \\
(7.8) \\
53 .\end{array}$ & $\begin{array}{c}0.04-0.07 \\
(0.03)\end{array}$ & $\begin{array}{c}0.15-0.23 \\
(0.08) \\
0.15\end{array}$ & $\begin{array}{c}0.16-0.20 \\
(0.4)\end{array}$ \\
\hline \multirow{4}{*}{ 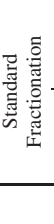 } & RWTD10 & $\begin{array}{l}\mathrm{KBE}=1.1 \\
\mathrm{RBE} \neq 1.1 \\
\mathrm{RBE}=1.1\end{array}$ & $\begin{array}{c}53.8 \\
94-139 \\
(45) \\
88.26 \\
\end{array}$ & $\begin{array}{c}14.1 \\
79-82 \\
(3) \\
84.2 \\
\end{array}$ & $\begin{array}{c}0.20 \\
74.9-77.2 \\
(2.3) \\
80.4 \\
\end{array}$ & $\begin{array}{c}47.6 \\
65-66 \\
(1) \\
67.5 \\
\end{array}$ & $\begin{array}{c}0.01 \\
31.1-31.6 \\
(0.5) \\
32.9 \\
\end{array}$ & $\begin{array}{c}0.01 \\
25-26.7 \\
(1.7) \\
25.6\end{array}$ & $\begin{array}{c}52.8 \\
56-71 \\
(15) \\
58.0\end{array}$ & $\begin{array}{c}53.2 \\
53-71 \\
(18) \\
57.7\end{array}$ & $\begin{array}{c}0.05 \\
52-72 \\
(20) \\
55.8\end{array}$ & $\begin{array}{c}0.15 \\
41-62 \\
(21) \\
43.9\end{array}$ & $\begin{array}{c}0.17 \\
39-59 \\
(20) \\
42\end{array}$ \\
\hline & \multirow[t]{3}{*}{ RWTD90 } & $\mathrm{RBE} \neq 1.1$ & $\begin{array}{c}91-133 \\
(42)\end{array}$ & $\begin{array}{l}22-24 \\
(2)\end{array}$ & $\begin{array}{c}0.31-0.34 \\
(0.03)\end{array}$ & $\begin{array}{c}61-62 \\
(1)\end{array}$ & 0.01 & 0.01 & $\begin{array}{l}52-57 \\
(5)\end{array}$ & $\begin{array}{c}52-69 \\
(17)\end{array}$ & 0.1 & $\begin{array}{c}0.1-0.2 \\
(0.1)\end{array}$ & $\begin{array}{c}0.1-0.2 \\
(0.1)\end{array}$ \\
\hline & & $\mathrm{RBE}=1.1$ & 85.7 & 20.7 & 0.32 & 63.7 & 0.01 & 0.01 & 55.5 & 55.9 & 0.07 & 0.2 & 0.16 \\
\hline & & & & Prostate & & & Liver & & & & Brain & & \\
\hline
\end{tabular}


Table 3. SD of the upper and lower bound of the RWTD ranges presented in table 2 for the standard number of fractionation.

\begin{tabular}{|c|c|c|c|c|c|c|c|c|c|c|c|c|}
\hline & & \multicolumn{3}{|c|}{ Liver } & \multicolumn{3}{|c|}{ Prostate } & \multicolumn{5}{|c|}{ Brain } \\
\hline & & GTV & Lung & $\begin{array}{l}\text { Healthy } \\
\text { liver }\end{array}$ & GTV & $\begin{array}{l}\text { Ant. } \\
\text { rectum }\end{array}$ & Bladder & GTV & Chiasm & Brainstem & $\begin{array}{l}\text { Lt opt. } \\
\text { nerv. }\end{array}$ & $\begin{array}{l}\text { Rt opt. } \\
\text { nerv. }\end{array}$ \\
\hline \multirow[t]{2}{*}{ Upper } & $10 \%$ & 4.982 & 7.534 & 23.065 & 2.071 & 9.439 & 3.026 & 4.736 & 6.302 & 3.749 & 12.090 & 13.491 \\
\hline & $90 \%$ & 1.695 & 0.018 & 0.007 & 2.576 & 5.396 & 0.349 & 2.606 & 8.147 & 0.119 & 0.182 & 0.230 \\
\hline \multirow[t]{2}{*}{ Center } & $10 \%$ & 6.631 & 10.073 & 22.737 & 4.352 & 5.308 & 2.947 & 5.566 & 7.932 & 3.212 & 14.041 & 11.346 \\
\hline & $90 \%$ & 1.085 & 0.019 & 0.007 & 4.504 & 3.772 & 0.267 & 3.999 & 7.620 & 0.121 & 0.243 & 0.235 \\
\hline \multirow[t]{2}{*}{ Lower } & $10 \%$ & 2.884 & 7.535 & 18.054 & 2.933 & 3.977 & 2.390 & 1.052 & 7.863 & 3.974 & 11.107 & 13.990 \\
\hline & $90 \%$ & 2.345 & 0.019 & 0.000 & 4.501 & 2.798 & 0.090 & 1.998 & 6.924 & 0.082 & 0.296 & 0.295 \\
\hline
\end{tabular}

are very similar, but because the physical dose received by the seminal vesicles is lower than the GTV one might expect a larger RBE in the seminal vesicles. The RBE values in the prostate are higher than for the seminal vesicles because tissues with lower $(\alpha / \beta)_{x}$, such as the prostate, the optic nerves and the brainstem, cause elevated RBE values.

The step in the RWTD-VH curve of the brain GTV at the $80 \%$ volume level in figure 3 is due to an overlap of the brainstem contour with the GTV contour. Because of the low $(\alpha / \beta)_{x}$ of the brainstem $(1.5 \mathrm{~Gy})$ compared to the GTV $(10 \mathrm{~Gy})$ we see an increase of the RWTD of the GTV. The higher $(\alpha / \beta)_{x}$ boundary of the RWTD-VH for the GTV is not equally affected because the high $(\alpha / \beta)_{x}$ cause a smaller variation in the already low $\mathrm{RBE}$ value.

According to table 2, the following features can be observed.

(1) The RWTD ranges become wider (larger RBE uncertainty) for low $(\alpha / \beta)_{x}$ and larger number of fractions (i.e. lower dose per fraction). This is particularly clear when comparing the three GTV columns in table 2, which shows the following.

(a) For prostate $(1.2 \mathrm{~Gy}<\alpha / \beta<5 \mathrm{~Gy})$ the average range of RWTD received by $90 \%$ $\left(\Delta \mathrm{RWTD}_{\mathrm{Avg}}^{90 \%}\right.$ ) of the GTV is $25 \mathrm{~Gy}(\mathrm{RWTD})$ (for the three fractionation schemes in table 2: $(42+21+12) / 3$ Gy(RWTD)) with a maximum of 42 Gy(RWTD) for standard number of fractions and a minimum of 12 Gy(RWTD) for hypofractionated treatments. The $10 \%$ of the GTV receives an average range of RWTD $\left(\Delta \mathrm{RWTD}_{\mathrm{Avg}}^{10 \%}\right)$ of 27 (45-13) Gy(RWTD).

(b) For brain (10 Gy $<\alpha / \beta<15 \mathrm{~Gy})$ : $\Delta$ RWTD $_{\text {Avg }}^{10 \%}=7.3(15-0.9)$ Gy(RWTD), $\triangle \mathrm{RWTD}_{\mathrm{Avg}}^{90 \%}=3.8(5-2.1) \mathrm{Gy}(\mathrm{RWTD})$.

(c) For liver (13 Gy $<\alpha / \beta<17 \mathrm{~Gy})$ : $\Delta$ RWTD $_{\mathrm{Avg}}^{10 \%}=0.7(1-0.4) \mathrm{Gy}(\mathrm{RWTD})$, $\Delta \mathrm{RWTD}_{\mathrm{Avg}}^{90 \%}=0.8(1-0.4) \mathrm{Gy}(\mathrm{RWTD})$.

(2) The RWTD uncertainty in the case of the rectum, bladder, lung and normal liver was found lower even for large uncertainties in the $(\alpha / \beta)_{x}$.

(a) $\operatorname{Rectum}(2.5 \mathrm{~Gy}<\alpha / \beta<5 \mathrm{~Gy})$ : $\Delta \mathrm{RWTD}_{\mathrm{Avg}}^{10 \%}=1.7(3-1) \mathrm{Gy}(\mathrm{RWTD})$, $\triangle \mathrm{RWTD}_{\mathrm{Avg}}^{90 \%}=1.3(2-1) \mathrm{Gy}(\mathrm{RWTD})$.

(b) Bladder (3 Gy $<\alpha / \beta<7$ Gy): $\Delta \mathrm{RWTD}_{\mathrm{Avg}}^{10 \%}=1.2(2.3-0.2) \mathrm{Gy}(\mathrm{RWTD})$, $\triangle \mathrm{RWTD}_{\mathrm{Avg}}^{90 \%}=0.01(0.03-0) \mathrm{Gy}(\mathrm{RWTD})$.

(c) Lung (1.6 Gy $<\alpha / \beta<6.9$ Gy): $\Delta \mathrm{RWTD}_{\mathrm{Avg}}^{10 \%}=0.4(0.5-0.2) \mathrm{Gy}(\mathrm{RWTD})$, 
PROSTATE (BIOL-DOSE)
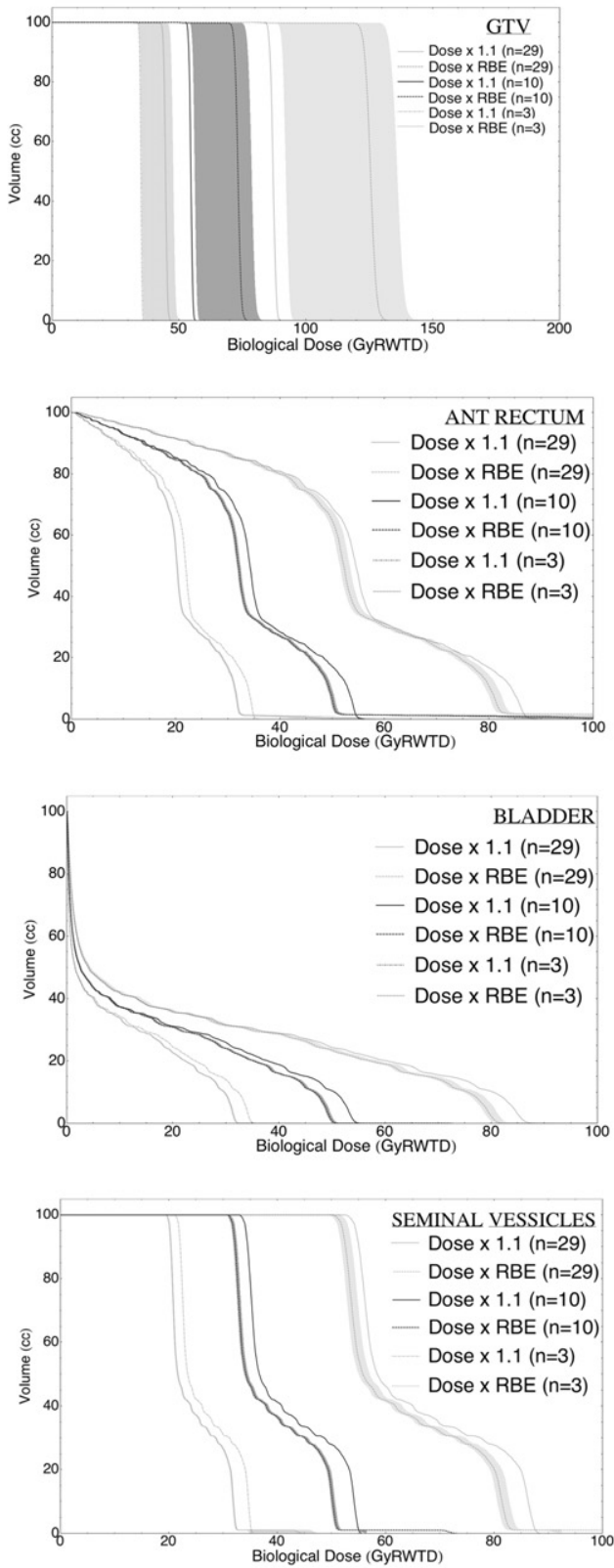

PROSTATE (LET \& RBE)
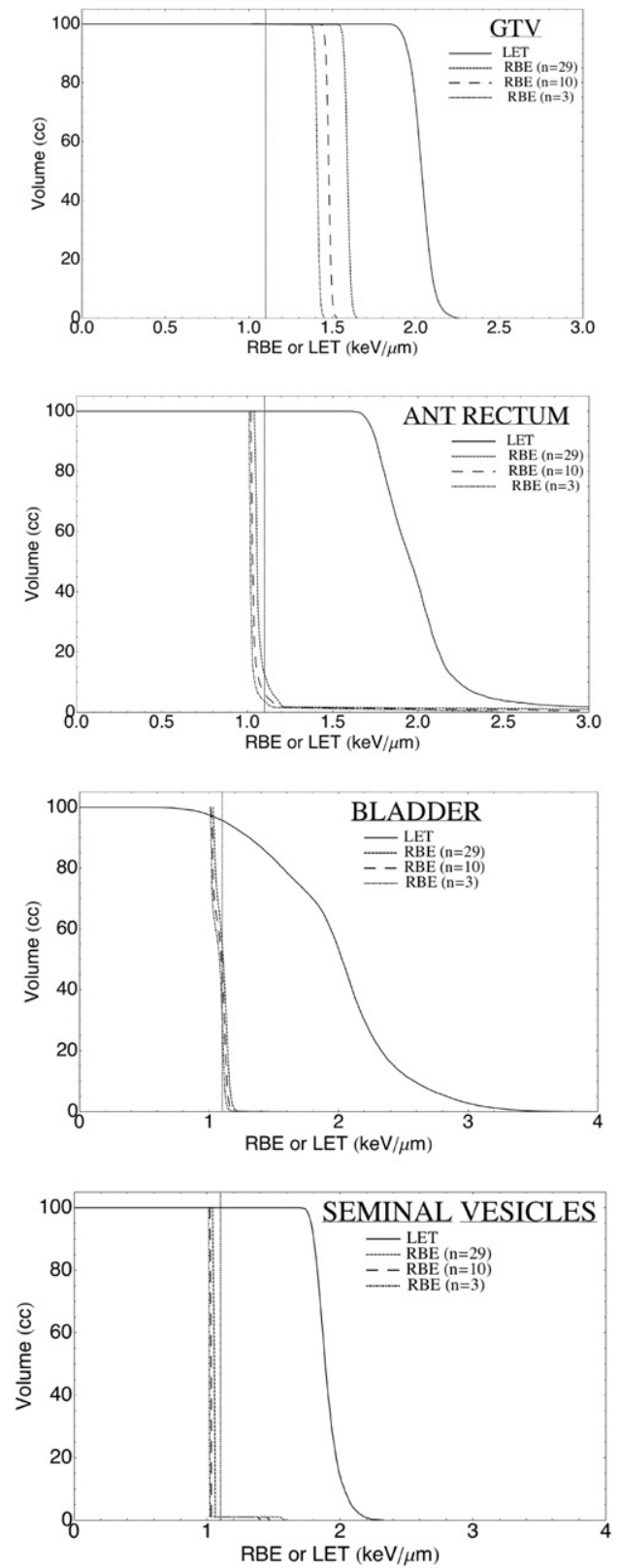

Figure 2. Results obtained as an average of five patients treated with protons for craniopharyngioma. ' $n$ ' represents the number of fractions. Left: DVHs of the RWTDs for the prostate GTV and the organs at risk. Each line is obtained from either the RWTD weighted by an RBE of 1.1 or a variable RBE that depends on dose, $\mathrm{LET}_{d}$ and $(\alpha / \beta)_{x}$. The gray bands correspond to the uncertainty of the RWTD due to the uncertainty of the tissues $(\alpha / \beta)_{x}$. Right: the RBE- and $\mathrm{LET}_{d}$-volume-histograms representing the distribution of $\mathrm{RBE}$ and $\mathrm{LET}_{d}$ calculated within each organ. The solid black line corresponds to the $\mathrm{LET}_{d}$, while the dashed lines correspond to the RBE for different doses per fraction. The vertical dashed line corresponds to a constant RBE of 1.1. 
BRAIN (BIOL-DOSE)
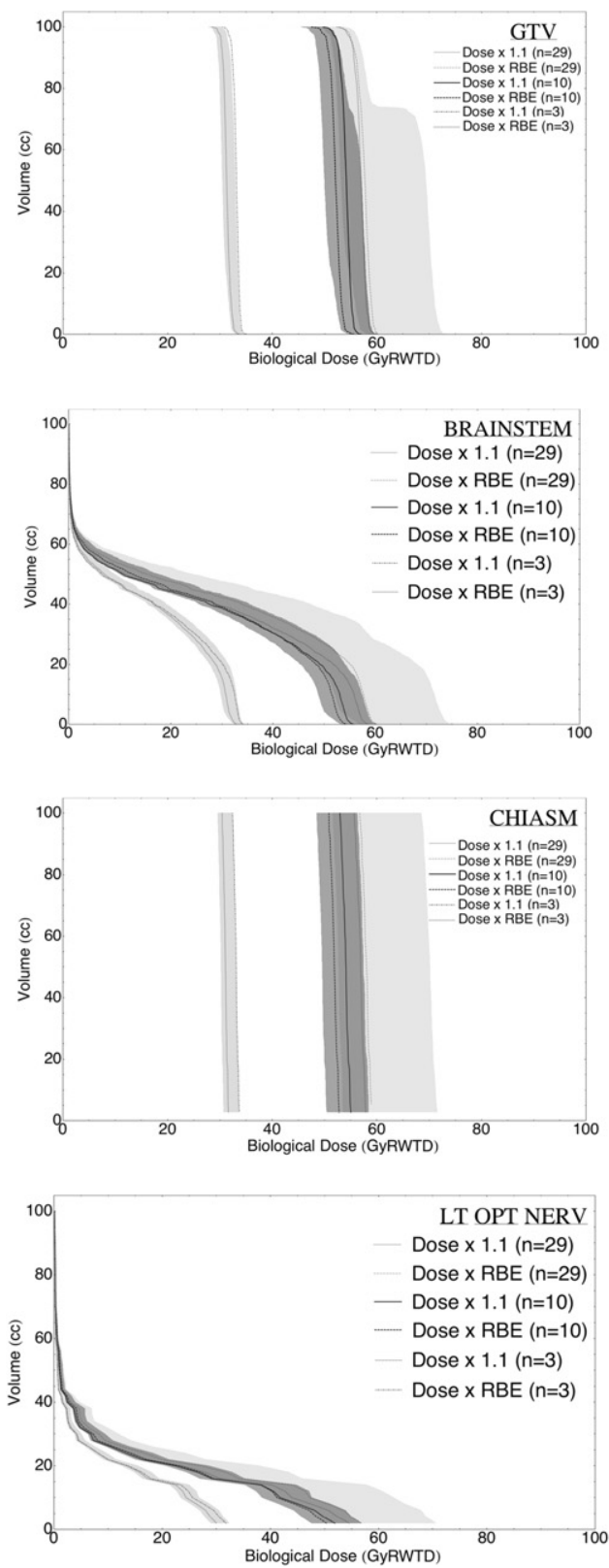

BRAIN (LET \& RBE)
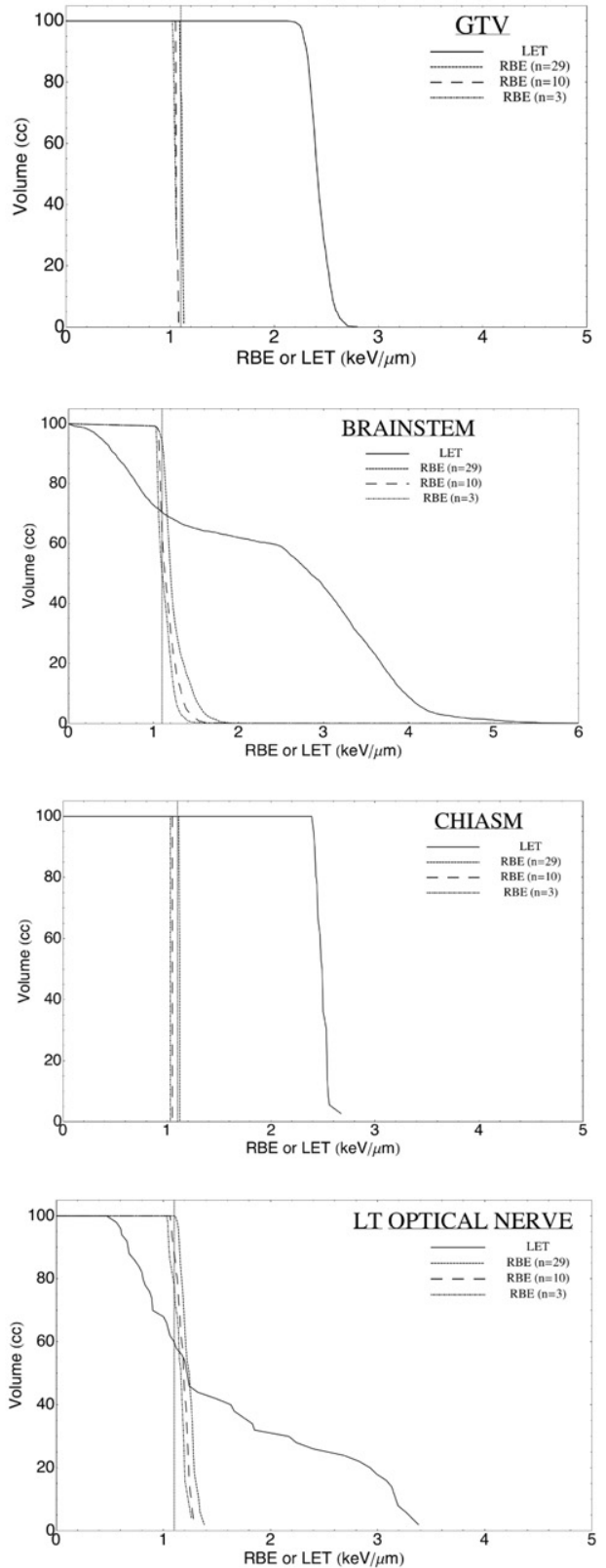

Figure 3. Results obtained as an average of five patients treated with protons for prostate cancer. ' $n$ ' represents the number of fractions. Left: DVHs of the RWTDs for the brain GTV and the organs at risk. Each line is obtained from either the RWTD weighted by an RBE of 1.1 or a variable RBE that depends on dose, $\mathrm{LET}_{d}$ and $(\alpha / \beta)_{x}$. The gray bands correspond to the uncertainty of the RWTD due to the uncertainty of the tissues $(\alpha / \beta)_{x}$. Right: the RBE- and LET $d$-volume-histograms representing the distribution of RBE and $\mathrm{LET}_{d}$ calculated within each organ. The solid black line corresponds to the $\mathrm{LET}_{d}$, while the dashed lines correspond to the RBE for different doses per fraction. The vertical dashed line corresponds to a constant RBE of 1.1. 
$\triangle$ RWTD $_{\text {Avg }}^{90 \%}=0.01 \mathrm{~Gy}(\mathrm{RWTD})$.

(d) Healthy liver (1.5 Gy $<\alpha / \beta<3$ Gy):

$\Delta \mathrm{RWTD}_{\mathrm{Avg}}^{10 \%}=1.0(1.7-0.2) \mathrm{Gy}(\mathrm{RWTD})$

$\triangle \mathrm{RWTD}_{\mathrm{Avg}}^{90 \%}=0 \mathrm{~Gy}(\mathrm{RWTD})$.

(3) Normal tissues with small $(\alpha / \beta)_{x}$ exposed to larger values of LET do present large RWTD uncertainties, such as in the case of the following.

(a) Chiasm (1.5 Gy $<\alpha / \beta<3.9$ Gy):

$\Delta$ RWTD $_{\mathrm{Avg}}^{10 \%}=9.6(18-2.8) \mathrm{Gy}(\mathrm{RWTD})$,

$\triangle \mathrm{RWTD}_{\mathrm{Avg}}^{90 \%}=9.2(17-2.8) \mathrm{Gy}(\mathrm{RWTD})$.

(b) Brainstem (1.5 Gy $<\alpha / \beta<3.9$ Gy):

$\triangle$ RWTD $_{\mathrm{Avg}}^{10 \%}=10.5(20-3) \mathrm{Gy}(\mathrm{RWTD})$,

$\triangle$ RWTD $_{\text {Avg }}^{90 \%}=0.01(0.01-0.03)$ Gy(RWTD).

(c) Optical nerves (averaged for both nerves) $(0.5 \mathrm{~Gy}<\alpha / \beta<10.3 \mathrm{~Gy}$ ):

$\triangle$ RWTD $_{\text {Avg }}^{10 \%}=11.2(21-0.01) \mathrm{Gy}(\mathrm{RWTD})$,

$\Delta$ RWTD $_{\text {Avg }}^{90 \%}=0.13(0.4-0.05)$ Gy(RWTD).

(4) The RWTD uncertainty for large doses (hypofractionated schemes) is smaller than for small doses (standard fractionation). This is corroborated by looking at figures 2 and 3 where the shaded areas of RWTD become narrower when going from standard fractionation to the hypofractionated schemes. As the total dose required in the hypofractionated regimes is lower, smaller uncertainties in the RWTD are expected.

(5) It seems that for $50 \%$ of the hypofractionated treatments in table 2 (22 cells out of 44 in the $n=10$ and $n=3$ rows), the RWTD for a constant RBE is larger than the RWTD range for a variable RBE, whereas for the remaining 50\% the RWTD for a constant RBE is within or below the RWTD range for a variable RBE. This could potentially imply that, when using hypofractionated schedules, one might have to decrease the dose required to maintain tumor control while reducing the entrance dose as well as the dose to critical organs.

\section{Discussion}

Biological optimization of proton treatments requires the consideration of the full range of RBE values across the SOBP as a function of $\mathrm{LET}_{d}$, dose and $(\alpha / \beta)_{x}$. However there is not sufficient data to calculate these values for all tissues as $(\alpha / \beta)_{x}$ is not precisely known for all tissues. The RBE value of 1.1 has proven to be a good average representation across the SOBP at any dose and for all tissues (Paganetti et al 2002). Nevertheless, if we want to increase the accuracy of our treatment to levels below 5-10\% of uncertainty, we need to consider biological effect variations that are above this level. Table 2 as well as figures 2 and 3 show in what circumstances (i.e. combination of $(\alpha / \beta)_{x}$ and/or doses per fraction) these levels of uncertainty are exceeded. According to table 2, the uncertainty levels of the RWTD (expressed as a percentage of the clinical biological dose: $1.1 \times$ Prescribed dose) due to the variability of the tissue $(\alpha / \beta)_{x}$ are above $10 \%$ uncertainty, with a maximum of $52.3 \%$ in the case of the prostate GTV (using the RWTD10 range for standard fractionation: Uncertainty $\left.(\%)=\frac{\mathrm{RWTD} \text { range }}{1.1 \times \text { Prescibed dose }} \times 100 \%=\frac{45(\mathrm{~Gy}(\mathrm{RWTD}))}{1.1 \times 78.3(\mathrm{~Gy})} \times 100 \%\right), 2.6 \%$ in the case of healthy liver (from the RWTD10 range in standard fractionation: $\frac{1.7(\mathrm{~Gy}(\mathrm{RWTD}))}{1.1 \times 60.2(\mathrm{~Gy})} \times 100 \%$ ). These percentages decrease for hypofractionated regimes.

The ranges of RWTD on the top of every cell in table 2 are due to the variability of $(\alpha / \beta)_{x}$, and the size of these ranges can be evaluated when using different fractionation schemes (or 
different dose levels). However, the dependence of RWTD with Let also has an important impact on how RWTD varies with $(\alpha / \beta)_{x}$ and dose per fraction, specially from the point of view that RBE increases with increasing LET but decreases with increasing $(\alpha / \beta)_{x}$ or dose. Thus, organs with large values of $(\alpha / \beta)_{x}$ receiving large values of LET may not present a large RWTD range. This effect can be seen in the case of the bladder, which according to figure 2 receives the highest values of LET in the case of prostate treatments, but this organ also has the largest values of $(\alpha / \beta)_{x}$ of this site, so that could potentially explain why the RWTD uncertainty for the bladder is small even for a large uncertainty in the $(\alpha / \beta)_{x}$ as explained in feature (2) of the results. The same reasoning could apply to the anterior rectum, which also receives higher LET than the GTV or seminal vesicles but the RWTD range is low even for large ranges of $(\alpha / \beta)_{x}$. As the $(\alpha / \beta)_{x}$ values related to the rectum are lower than those for the bladder larger RWTD ranges are observed. As the rectum and the bladder are symmetrically placed on the sides of the prostate, it could be assumed that they both receive a similar dose, so the above discussion is independent of dose differences received by both organs. Also, within the discussion of competing effects between LET and $(\alpha / \beta)_{x}$ on RWTD, it is interesting to note that, in the case of the prostate GTV where the LET decreases to values just above $2 \mathrm{keV} \mu \mathrm{m}^{-1}$ but the lower bound of the $(\alpha / \beta)_{x}$ range goes as low as $1.2 \mathrm{~Gy}$, a larger decrease of LET would have been required to compensate for such low values of $(\alpha / \beta)_{x}$ in order to have small variations of RWTD. This feature clearly shows how important is the impact on RBE due to variations of $(\alpha / \beta)_{x}$ versus variations of LET.

According to figure 1, the critical structures in the brain are subjected to a rapid decrease of dose (dose falloff), and increased LET and very large ranges of $(\alpha / \beta)_{x}$ going from 0.5 to $10 \mathrm{~Gy}$. According to what has been discussed above, and considering that in this case all three factors (dose, LET and $(\alpha / \beta)_{x}$ ) work in the same direction to increase RBE, it is not surprising to find the largest RWTD ranges overall in this site. This shows how, in the case of brain treatments, radiobiological considerations in proton treatment planning are of special importance compared to any other treatment site. In the case of the liver, the larger RWTD ranges are observed in the healthy liver where again the low $(\alpha / \beta)_{x}$, the increased LET values (as the beams stop in the healthy liver in most of the cases) and the dose falloff imply larger values of RBE. The ranges are however not very large in the healthy liver due to the small range of $(\alpha / \beta)_{x}$.

The correctness of these conclusions and the results of the present analysis is based on the validity of equations (2) and (3), but there is not sufficient data to either confirm or refute their validity. The large uncertainties discovered in the case of the prostate and the brain, imply that more data are required before considering correcting for RBE effects. For this reason, a better approach in this case would be to use a beam arrangement that allows placing the high LET components of the beam away from the critical structures (Grassberger et al 2011). This was not feasible for the planning and delivery scenario considered in this study because we considered passive scattering treatments. Note also that an unaccounted increase of RBE in the distal falloff can cause the extension of the distal edge of the biological dose by a few millimeters (Robertson et al 1975, Carabe et al 2012).

As part of the discussion on the uncertainty of the RBE values derived in this work, we need to refer to the questionable validity of the LQ model (and therefore of equation (1)) for the doses per fraction of the hypofractionated regimens used here. The largest dose used in the calculations of the RBE was $11.5 \mathrm{~Gy}$ in the case of the liver (34.4 Gy delivered in three fractions). However, there are studies (Brenner 2008) suggesting the validity of the LQ at doses as high as 18 Gy of photons but no equivalent value has been suggested for protons (although it is assumed that it will be lower due to the high-LET components in a proton beam). It is therefore difficult to quantify the uncertainty induced by this limitation in the LQ model. 

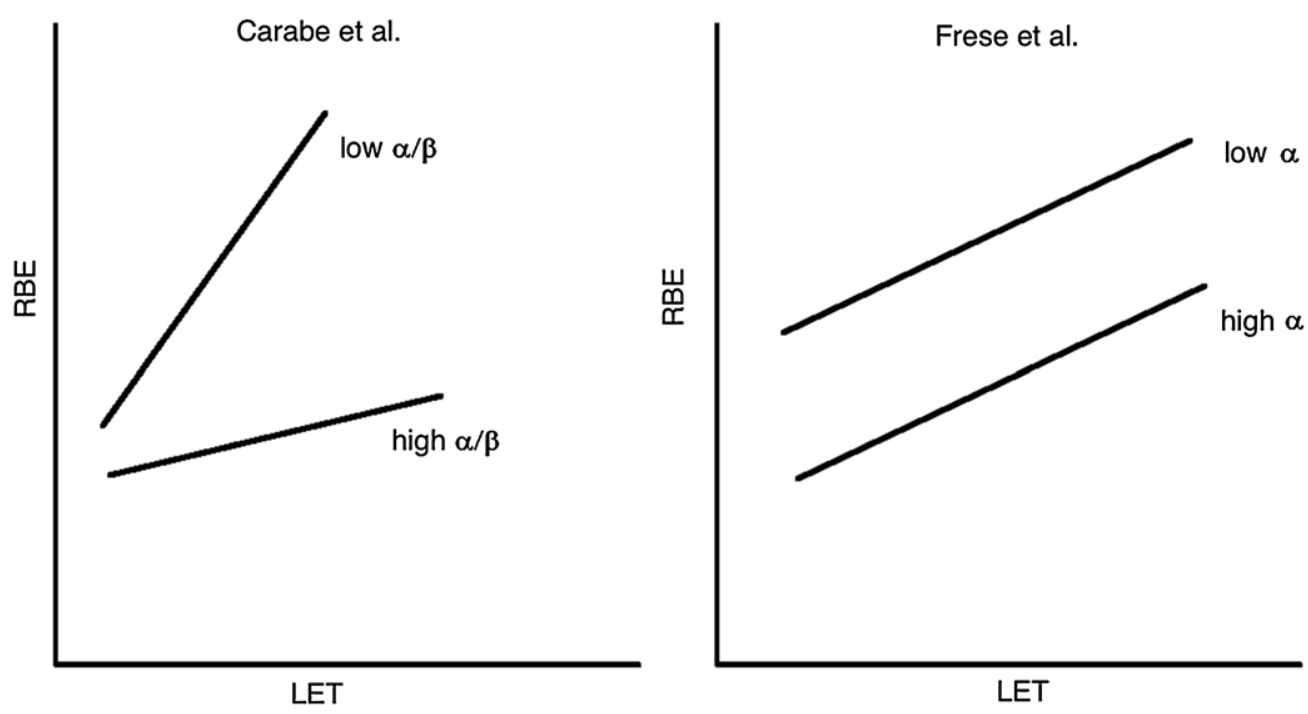

Figure 4. Difference in the interpretation of how the relationship between RBE and LET should change with the LQ model parameters by Frese et al (2011) and Carabe-Fernandez et al (2007).

The impact of variable RBE values in proton therapy has been modeled before (Tilly et al 2005, Frese et al 2011). Tilly et al found that the ratio of $\alpha_{\text {proton }} / \alpha_{x}$ (equals $\mathrm{RBE}_{\max }$ in this work) linearly increases with $\mathrm{LET}_{d}$, and that the slope of this linear relationship increases with decreasing $(\alpha / \beta)_{x}$. However, in their study, the linear relationship between $\alpha_{\text {proton }} / \alpha_{x}$ and $\operatorname{LET}_{d}$ was established based on only two values of $(\alpha / \beta)_{x}(2$ and $10 \mathrm{~Gy})$ and a sensitivity analysis of the change of slope was performed by providing a maximum and minimum slope corresponding to the estimated error associated with the experimental values of $(\alpha / \beta)_{x}$. Thus, the analysis was performed for tissues with $(\alpha / \beta)_{x}$ equal or around 2 and $10 \mathrm{~Gy}$. In contrast, our analysis handles the change of slope with $(\alpha / \beta)_{x}$ as part of the model input parameters $\mathrm{RBE}_{\max }$ and $\mathrm{RBE}_{\min }$. Our approach will allow us to incorporate additional experimental data for $(\alpha / \beta)_{x}$ values, if they become available. Furthermore, the inclusion of $\mathrm{RBE}_{\min }$ in equation (1) makes our model suitable to predict RBE effects in hypofractionated regimens.

The model by Frese et al (2011) is similar to equation (1) with the difference that it assumes a constant $\beta_{x}$ as a function of $\mathrm{LET}_{d}$. The model does consider a change of the slope of the relationship between $\alpha_{\text {protons }}$ and $\mathrm{LET}_{d}$ via the parameter $\lambda: \alpha_{\text {protons }}=\alpha_{\mathrm{o}}+\lambda \cdot \mathrm{LET}_{d}$, where $\alpha_{\mathrm{o}}$ corresponds to a normalization parameter such that in the entrance of the proton beam the RBE equals 1.0. The parameter $\lambda$ is independent of the biological tissue and the tissue dependence is solely described by $\alpha_{0}$, which depends on $\alpha_{x}$. The difference to our approach is illustrated in figure 4. A relationship similar to the one proposed in our work has been proposed by others (Hawkins 1994, 2003). In contrast to the model by Frese et al one might assume that in the case of a cell or tissue with a low $\alpha_{x}$, low $\mathrm{LET}_{d}$ values will not necessarily yield large values of RBE according to our model. Furthermore, the approach by Frese et al relies on the knowledge of $\alpha_{x}$ instead of $(\alpha / \beta)_{x}$ alone.

As we have shown, considering variations in the biological effect of proton beams might offer the potential to further optimize proton therapy treatments. Furthermore, biological considerations beyond simply using an RBE of 1.1 might be warranted when comparing photon and proton treatments in clinical trials, in particular for tissues with low $(\alpha / \beta)_{x}$ ratios, such as prostate. 


\section{Acknowledgments}

The project was supported by the Federal Share of program income earned by Massachusetts General Hospital on C06 CA059267, Proton Therapy Research and Treatment Center. Thanks are extended to Professor Roger G Dale for his useful comments on the BED calculations.

\section{References}

Amols H I and Kliauga P 1985 Microdosimetry of 10-18 MeV electrons and photons using walled and wall-less detectors Radiat. Prot. Dosim. 13 365-8

Belli M et al 1989 RBE-LET relationship for the survival of V79 cells irradiated with low energy protons Int. J. Radiat. Biol. 55 93-104

Belli M et al 1993 Inactivation and mutation induction in V79 cells by low energy protons: re-evaluation of the results at the LNL facility Int. J. Radiat. Biol. 3 331-7

Brenner D J 2008 The linear-quadratic model is an appropriate methodology for determining isoeffective doses at large doses per fraction Semin. Radiat. Oncol. 18 234-9

Brenner D J and Hall E J 1999 Fractionation and protraction for radiotherapy of prostate carcinoma Int. J. Radiat. Oncol. Biol. Phys. 43 1095-101

Carabe A 2013 Radiobiology of proton and carbon ion therapy Proton and Carbon Therapy (Imaging Medical Diagnosis and Therapy Series) ed C M Charlie Ma and T Lomax (Boca Raton, FL: CRC Press)

Carabe A et al 2012 Range uncertainty in proton therapy due to variable biological effectiveness Phys. Med. Biol. 57 1159-72

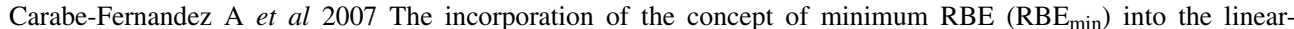
quadratic model and the potential for improved radiobiological analysis of high-LET treatments Int. J. Radiat. Biol. 83 27-39

Carabe-Fernandez A et al 2010 Fractionation effects in particle radiotherapy: implications for hypo-fractionation regimes Phys. Med. Biol. 55 5685-700

Coutrakon $\mathrm{G}$ et al 1997 Microdosimetry spectra of the Loma Linda proton beam and relative biological effectiveness comparisons Med. Phys. 24 1499-506

Dale R G et al 2002 Practical methods for compensating for missed treatments days in radiotherapy, with particular reference to head and neck schedules Clin. Oncol. 14 382-93

Daşu A 2007 Is the alpha/beta value for prostate tumours low enough to be safely used in clinical trials? Clin. Oncol. (R. Coll. Radiol.) 19 289-301

Folkard M et al 1996 Inactivation of V79 cells by low-energy protons, deuterons and helium-3 ions Int. J. Radiat. Biol. 69 729-38

Fowler J F 1989 The linear-quadratic formula and progress in fractionated radiotherapy Br. J. Radiol. 62 679-94

Fowler J F 2005 The radiobiology of prostate cancer including new aspects of fractionated radiotherapy Acta Oncol. $44265-76$

Frese M C et al 2011 Application of constant vs. variable relative biological effectiveness in treatment planning of intensity modulated proton therapy Int. J. Radiat. Oncol. Biol. Phys. 79 80-8

Gerweck L E and Kozin S V 1999 Relative biological effectiveness of proton beams in clinical therapy Radiother. Oncol. 50 135-42

Grassberger C et al 2011 Variations in linear energy transfer within clinical proton therapy fields and the potential for biological treatment planning Int. J. Radiat. Oncol. Biol. Phys. 80 1559-66

Hawkins R B 1994 A statistical theory of cell killing by radiation of varying linear energy transfer Radiat. Res. 140 3366-74

Hawkins R B 2003 A microdosimetric-kinetic model for the effect of non-Poisson distribution of lethal lesions on the variation of RBE with LET Radiat. Res. $16061-9$

Jiang G L et al 1994 Radiation-induced injury to the visual pathway Radiother. Oncol. 30 17-25

Jones B, Underwood T S A, Carabe-Fernandez A, Timlin C and Dale R G 2011 Fast neutron relative biological effects and implications for charged particle therapy Br. J. Radiol. 84 S11-8

Koukourakis M I et al 2007 Biological dose volume histograms during conformal hypofractionated accelerated radiotherapy for prostate cancer Med. Phys. 34 76-80

Lawrence YR et al 2010 Radiation dose-volume effects in the brain Int. J. Radiat. Oncol. Biol. Phys. 76 (3 Suppl) S20-7

Meeks S L et al 2000 Calculation of cranial nerve complication probability for acoustic neuroma radiosurgery Int. J. Radiat. Oncol. Biol. Phys. 47 597-602 
Paganetti H et al 2000 The general relation between tissue response to x-radiation ( $\alpha / \beta$-values) and the relative biological effectiveness (RBE) of protons: prediction by the Katz track-structure model Int. J. Radiat. Biol. 76 985-98

Paganetti H et al 2002 Relative biological effectiveness (RBE) values for proton beam therapy Int. J. Radiat. Oncol. Biol. Phys. 53 407-21

Paganetti H et al 2008 Clinical implementation of full Monte Carlo dose calculation in proton beam therapy Phys. Med. Biol. 3 4825-53

Perris A et al 1986 Biological effectiveness of low energy protons: I. Survival of Chinese hamster cells Int. J. Radiat. Biol. 50 1093-101

Ritter M et al 2009 Hypofractionation for prostate cancer Cancer J. 15 1-6

Robertson J B et al 1975 Radiobiological studies of a high-energy modulated proton beam utilizing cultured mammalian cells Cancer 35 1664-77

Schettino G et al 2001 Low-dose hypersensitivity in Chinese hamster V79 cells targeted with counted protons using a charged-particle microbeam Radiat. Res. $156526-34$

Tai A et al 2008 Estimate of radiobiologic parameters from clinical data for biologically based treatment planning for liver irradiation Int. J. Radiat. Oncol. Biol. Phys. 70 900-7

The Royal College of Radiologists 2008 The Timely Delivery of Radical Radiotherapy: Standards and Guidelines for the Management of Unscheduled Treatment Interruptions 3rd edn (London: The Royal College of Radiologists)

Tilly N et al 2005 The influence of RBE variations in a clinical proton treatment plan for a hypopharynx cancer Phys. Med. Biol. $502765-77$

Wilkens J J and Oelfke U 2004 A phenomenological model for the relative biological effectiveness in therapeutic proton beams Phys. Med. Biol. 49 2811-25

Wouters B G et al 1996 Measurements of relative biological effectiveness of the $70 \mathrm{MeV}$ proton beam at TRIUMF using Chinese hamster V79 cells and the high-precision cell sorter assay Radiat. Res. 146 159-70

Yuan J et al 2008 Hypofractionation regimens for stereotactic radiotherapy for large brain tumors Int. J. Radiat. Oncol. Biol. Phys. 72 390-7 